\title{
AUTOMATIC CONTINUITY OF PERTURBATIONS OF CAUSAL OPERATORS
}

\author{
K.J. Harrison, L-J. Eaton and J.A. Ward
}

\begin{abstract}
We obtain automatic continuity results for finite-rank perturbations of causal sequence space operators, and provide examples to illustrate cases where automatic continuity does not hold.
\end{abstract}

\section{INTRODUCTION}

Let $\mathcal{E}$ be a Banach space and let $\Xi(=\Xi(\mathcal{E}))$ denote the linear space of all doubly infinite $\mathcal{E}$-valued sequences. For each $p, \ell^{p}\left(=\ell^{p}(\mathcal{E})\right)$ is the Banach space consisting of all $\mathcal{E}$-valued sequences $x \in \Xi$ for which $\|x\|_{p}<\infty$, where $\|x\|_{p}=\left(\sum_{n}\left\|x_{n}\right\|^{p}\right)^{1 / p}$ for $1 \leqslant p<\infty,\|x\|_{\infty}=\sup _{n}\left\|x_{n}\right\|$, and where $\left\|x_{n}\right\|$ is the norm of $x_{n}$ in the base space $\mathcal{E}$.

For each integer $k$, we introduce the projection $P_{k}$ on $\Xi$, defined by $\left(P_{k} x\right)_{n}=x_{n}$ if $n \leqslant k$ and $\left(P_{k} x\right)_{n}=0$ otherwise. We say that a sequence $x \in \Xi$ has a finite past if and only if $P_{k} x=0$ for some integer $k$, and in particular that $x$ is causal if $P_{-1} x=0$ so that $x_{n}=0$ if $n<0$. We let $\Xi_{f p}$ denote the set of all sequences in $\Xi$ with finite past, $\Xi_{+}$the set of all causal sequences and also $\Xi_{00}$ the set of all sequences $x$ with a finite support; that is, for which $x_{n}=0$ off some finite subset of $\mathbf{Z}$.

A sequence space operator is a linear operator between subspaces of $\Xi$. We are interested in the relationship between the notions of stability and continuity for sequence space operators which arise naturally using the $\ell^{p}$ subspaces.

Let $T$ be a sequence space operator and let $\mathcal{W}$ be a subspace of $\Xi$. We say that $T$ is $(p, q)$-stable on $\mathcal{W}$ if $\mathcal{W} \cap \ell^{p} \subseteq \mathcal{D}(T)$, the domain of $T$, and $T x \in \ell^{q}$ for each $x \in \mathcal{W} \cap \ell^{p}$. We say that $T$ is $(p, q)$-stable if it is $(p, q)$-stable on $\ell^{p}$. Operators which are $(\infty, \infty)$-stable have been considered extensively in the signal processing literature, where they are said to be bounded-in-bounded-out.

We say that $T$ is $(p, q)$-continuous on $\mathcal{W}$ if it is $(p, q)$-stable on $\mathcal{W}$ and there is a number $\kappa \geqslant 0$ such that $\|T x\|_{q} \leqslant \kappa\|x\|_{p}$ for all $x$ in $\mathcal{W} \cap \ell^{p}$. The least such number $\kappa$ is called the $(p, q)$-operator norm of $T$ on $\mathcal{W}$. We say that $T$ is $(p, q)$-continuous

Received 15th April, 1996.

Copyright Clearance Centre, Inc. Serial-fee code: 0004-9729/97 \$A2.00+0.00. 
if it is $(p, q)$-continuous on $\ell^{p}$. Operators which are $(\infty, \infty)$-continuous have also been considered extensively in the signal processing literature, where they are said to have finite gain.

Clearly $(p, q)$-continuity on $\mathcal{W}$ implies $(p, q)$-stability on $\mathcal{W}$, but the converse is not true in general. In this paper, we shall be concerned with conditions on $T$ that guarantee that the reverse automatic continuity result holds when $\mathcal{W}$ is one of the spaces $\Xi_{+}, \Xi_{f p}$ or $\Xi$.

\section{OpERators on CAUSAL SEQUENCES}

2.1 CaUsal operators. A sequence space operator $C$ is causal on a subspace $\mathcal{W}$ of $\Xi$ if $\mathcal{W} \subseteq \mathcal{D}(C)$ and if, for each $x \in \mathcal{W}$ and each integer $k, P_{k} x \in \mathcal{D}(C)$ and $P_{k} C P_{k} x=P_{k} C x$. Thus $C$ is causal on $\mathcal{W}$ if, for any two sequences $x$ and $y$ in $\mathcal{W}$, the images $C x$ and $C y$ agree at the $k^{t h}$ coordinate if $x$ and $y$ agree at the $k^{t h}$ and all preceding coordinates.

Our starting point is a version of a well-known automatic continuity result for causal operators. See, for example, [4].

PROPOSITION 1. If $C$ is causal and $(p, q)$-stable on $\ell^{p}(\mathcal{E})_{+}=\ell^{p}(\mathcal{E}) \cap \Xi_{+}$, where $\mathcal{E}$ is finite dimensional and $1 \leqslant p, q \leqslant \infty$, then $C$ is $(p, q)$-continuous on $\ell^{p}(\mathcal{E})_{+}$.

PRoof: The neatest proof uses the closed graph theorem. Suppose that $x^{(n)} \rightarrow x$ in $\ell^{p}(\mathcal{E})_{+}$and $C x^{(n)} \rightarrow y$ in $\ell^{q}(\mathcal{E})$, and choose any $k \geqslant 0$. Then

$$
P_{k} C x^{(n)}=P_{k} C P_{k} x^{(n)} \rightarrow P_{k} C P_{k} x=P_{k} C x
$$

because $C$ is causal and $P_{k} C$ is continuous on the finite-dimensional space $P_{k} \ell^{p}(\mathcal{E})_{+}$. But $P_{k} C x^{(n)} \rightarrow P_{k} y$, and so $P_{k} y=P_{k} C x$. Since $k$ is arbitrary, it follows that $y=C x$, and so $C$ is $(p, q)$-continuous on $\ell^{p}(\mathcal{E})_{+}$by the closed graph theorem.

Proposition 1 fails if $\mathcal{E}$ is infinite dimensional. To see this, let $\varphi$ be a discontinuous linear functional on $\mathcal{E}$, let $u$ be a non-zero sequence in $\ell^{q}(\mathcal{E})_{+}$, let $Q_{0}=P_{0}-P_{-1}$, and define $C$ on $\Xi$ by $C x=\varphi\left(Q_{0} x\right) u$. Then $C$ is $(p, q)$-stable, but clearly not $(p, q)$ continuous on $\Xi_{+}$. To see that $C$ is causal observe that, for any $x \in \ell^{p}(\mathcal{E})_{+}$and any integer $k$,

$$
P_{k} C P_{k} x=\varphi\left(Q_{0} P_{k} x\right) P_{k} u, \text { and } P_{k} C x=\varphi\left(Q_{0} x\right) P_{k} u .
$$

Since $Q_{0} P_{k}=Q_{0}$ if $k \geqslant 0$ and $P_{k} u=0$ if $k<0$, it follows that $P_{k} C P_{k} x=P_{k} C x$ for all $x$ and $k$. So $C$ is causal on $\Xi_{+}$.

The following result generalises Proposition 1. It concerns the continuity of causal operators which are 'almost' $(p, q)$-stable on $\ell^{p}(\mathcal{E})_{+}$. 
THEOREM 1. Suppose that $C$ is causal on $\ell^{p}(\mathcal{E})_{+}$and that $C \ell^{p}(\mathcal{E})_{+} \subseteq \ell^{q}(\mathcal{E})+\mathcal{V}$, where $\mathcal{E}$ is finite dimensional and $1 \leqslant p, q \leqslant \infty$, and where $\mathcal{V}$ is a finite-dimensional subspace of $\Xi$. Let $\mathcal{X}$ denote the stability set of $C$, that is,

$$
\mathcal{X}=C^{-1} \ell^{q}(\mathcal{E})=\left\{x: x \in \ell^{p}(\mathcal{E})_{+} \text {and } C x \in \ell^{q}(\mathcal{E})\right\} .
$$

Then $\mathcal{X}$ is a closed subspace of finite codimension in $\ell^{p}(\mathcal{E})_{+}$, and $C$ is $(p, q)$-continuous on $\mathcal{X}$.

PROOF: Let $m$ be a bounded sequence of non-zero numbers with the property that $m x \in \ell^{q}(\mathcal{E})$ for each $x \in \mathcal{V}$, and let $M$ be the sequence space operator defined by pointwise multiplication by $m$; that is $(M y)_{n}=m_{n} y_{n}$ for each integer $n$ and each $y$ in $\Xi$. Then $M$ is injective and causal, and $M y \in \ell^{q}(\mathcal{E})$ for each $y$ in $\ell^{q}(\mathcal{E})+\mathcal{V}$. Therefore $M C x \in M\left(\ell^{q}(\mathcal{E})+\mathcal{V}\right) \subseteq \ell^{q}(\mathcal{E})$ for each $x \in \ell^{p}(\mathcal{E})_{+}$, and so $M C$ is $(p, q)$ stable on $\ell^{p}(\mathcal{E})_{+}$. Furthermore, since $M$ is causal on $\Xi$ and $C$ is causal on $\ell^{p}(\mathcal{E})_{+}$, $M C$ is causal on $\ell^{p}(\mathcal{E})_{+}$. So by Proposition $1, M C$ is $(p, q)$-continuous on $\ell^{p}(\mathcal{E})_{+}$.

Let \|\| be a norm on $\ell^{q}(\mathcal{E})+\mathcal{V}$ with the property that

$$
\|y\|_{q} \leqslant\|y\| \text { for any } y \in \ell^{q}(\mathcal{E}),
$$

and suppose that $x^{(n)} \rightarrow x$ in $\ell^{p}(\mathcal{E})_{+}$and $C x^{(n)} \rightarrow y$ in $\ell^{q}(\mathcal{E})+\mathcal{V}$. Since $M C$ is $(p, q)$-continuous on $\ell^{p}(\mathcal{E})_{+}, M C x^{(n)} \rightarrow M C x$ in $\ell^{q}(\mathcal{E})$. But $M$ is also continuous as an operator from $\ell^{q}(\mathcal{E})+\mathcal{V}$ into $\ell^{q}(\mathcal{E})$. So $M C x^{(n)} \rightarrow M y$. Therefore $M y=M C x$, and since $M$ is injective, $y=C x$. So by the closed graph theorem, $C$ is continuous as an operator from $\ell^{p}(\mathcal{E})_{+}$into $\ell^{q}(\mathcal{E})+\mathcal{V}$. Therefore there exists $\kappa \geqslant 0$ such that $\|C x\| \leqslant \kappa\|x\|_{p}$ for all $x \in \ell^{p}(\mathcal{E})_{+}$, and it follows from (2) that $\|C x\|_{q} \leqslant\|C x\| \leqslant \kappa\|x\|_{p}$ for each $x \in \mathcal{X}$. So $C$ is $(p, q)$-continuous on $\mathcal{X}$.

Let $\left\{C x_{1}, C x_{2}, \ldots, C x_{k}\right\}$ be a basis of $\operatorname{ran}(C) \cap \mathcal{V}$, where each $x_{j} \in \ell^{p}(\mathcal{E})_{+}$, and choose any $x \in \ell^{P}(\mathcal{E})_{+}$. Then there exist scalars $\alpha_{1}, \alpha_{2}, \ldots, \alpha_{k}$ and $y \in \ell^{q}(\mathcal{E})$ such that $C x=y+\sum_{j=1}^{k} \alpha_{j} C x_{j}$. But this implies that $x-\sum_{j=1}^{k} \alpha_{j} x_{j} \in \mathcal{X}$. So $\mathcal{X}$ has finite-codimension in $\ell^{p}(\mathcal{E})_{+}$.

Finally, suppose that $x^{(n)} \rightarrow x$ in $\ell^{p}(\mathcal{E})_{+}$and that $x^{(n)} \in \mathcal{X}$ for each $n$. Since $C$ is causal and $P_{k} C$ is $(p, p)$-continuous on $P_{k} \ell^{p}(\mathcal{E})_{+}$, for each $k$,

$$
P_{k} C x^{(n)}=P_{k} C P_{k} x^{(n)} \rightarrow P_{k} C P_{k} x=P_{k} C x .
$$

So $C x^{(n)} \rightarrow C x$ coordinatewise. But $C$ is $(p, q)$-continuous on $\mathcal{X}$ and $\left\{\left\|x^{(n)}\right\|_{p}\right\}$ is bounded, and so $\left\{\left\|C x^{(n)}\right\|_{q}\right\}$ is bounded. Therefore $\|C x\|_{q}<\infty$, that is, $x \in \mathcal{X}$. So $\mathcal{X}$ is closed and the theorem is proved. 
2.2 Finite rank perturbations of Causal operators. In this section we use Theorem 1 to establish continuity results for finite-rank perturbations of causal operators.

Theorem 2. Suppose that $C$ is causal and $T$ is $(p, q)$-stable on $\ell^{p}(\mathcal{E})_{+}$, where $\mathcal{E}$ is finite dimensional and $1 \leqslant p, q \leqslant \infty$, and suppose that $\operatorname{rank}(T-C)=d<\infty$. Then there exists an operator $T^{\prime}$ which is $(p, q)$-continuous on $\ell^{p}(\mathcal{E})_{+}$and which satisfies $\operatorname{rank}\left(T^{\prime}-T\right) \leqslant d$ and $\operatorname{rank}\left(T^{\prime}-C\right) \leqslant d$.

Proof: Since $T$ is $(p, q)$-stable on $\ell^{p}(\mathcal{E})_{+}$and $F=T-C$ has finite rank, $C$ satisfies the conditions of Theorem 1 . So $C$ is $(p, q)$-continuous on its stability set $\mathcal{X}$, as defined in (1), and $\mathcal{X}$ is a closed subspace of finite codimension in $\ell^{p}(\mathcal{E})_{+}$. Let $\mathcal{Y}$ be a subspace which is complementary to $\mathcal{X}$ in $\ell^{p}(\mathcal{E})_{+}$, and let $T^{\prime}$ Be defined on $\ell^{p}(\mathcal{E})_{+}$ by

$$
T^{\prime}(x+y)=C x+T y \text { for each } x \in \mathcal{X} \text { and each } y \in \mathcal{Y} .
$$

Since $C$ is $(p, q)$-continuous on $\mathcal{X}$ and since $\mathcal{Y}$ is finite-dimensional, $T^{\prime}$ is $(p, q)$ continuous on $\ell^{p}(\mathcal{E})_{+}$. Furthermore,

$$
\left(T^{\prime}-T\right)(x+y)=-F x \text { and }\left(T^{\prime}-C\right)(x+y)=F x,
$$

and so $\operatorname{rank}\left(T^{\prime}-T\right) \leqslant d$ and $\operatorname{rank}\left(T^{\prime}-C\right) \leqslant d$, as required.

EXAmple 1. Suppose that $T$ and $C$ are defined on $\ell^{p}(\mathcal{E})_{+}$by

$$
T x=\psi(x) u \text { and } C x=\phi\left(Q_{0} x\right) v,
$$

where $u$ and $v$ are causal sequences with $\|u\|_{q}<\infty$ and $\|v\|_{q}=\infty$, where $\phi$ is a non-zero linear form on $\mathcal{E}$ and where $\psi$ is a discontinuous linear form on $\ell^{p}(\mathcal{E})_{+}$. Then $T$ is $(p, q)$-stable and $C$ is causal on $\ell^{p}(\mathcal{E})_{+}, T-C$ has rank 2 , and neither $T$ nor $C$ is $(p, q)$-continuous on $\ell^{p}(\mathcal{E})_{+}$. However the zero operator is a finite-rank perturbation of both $T$ and $C$ which is $(p, q)$-continuous on $\ell^{p}(\mathcal{E})_{+}$.

The following corollary gives a condition under which a $(p, q)$-stable finite-rank perturbation of a causal operator on $\ell^{p}(\mathcal{E})_{+}$is itself $(p, q)$-continuous.

COROLlaRY 1. Suppose that $C$ is causal and $T$ is $(p, q)$-stable on $\ell^{p}(\mathcal{E})_{+}$, where $\mathcal{E}$ is finite dimensional and $1 \leqslant p, q \leqslant \infty$. Suppose also that $T-C$ has finite rank, and that $(T-C) \ell^{p}(\mathcal{E})_{+} \cap \ell^{q}(\mathcal{E})=\{0\}$. Then $T$ is $(p, q)$-continuous on $\ell^{p}(\mathcal{E})_{+} \cdot$

Proof: If $x \in \mathcal{X}$, then $C x \in \ell^{q}(\mathcal{E})$. Now $T x \in \ell^{q}(\mathcal{E})$ because $T$ is $(p, q)$-stable on $\ell^{p}(\mathcal{E})_{+}$, and therefore $(T-C) x \in(T-C) \ell^{p}(\mathcal{E})_{+} \cap \ell^{q}(\mathcal{E})$. So by the hypothesis, $T x=C x$. Therefore $T=T^{\prime}$, where $T^{\prime}$ is defined as in (3), and the result now follows from Theorem 2. 
According to Proposition 1, a causal operator on $\ell^{p}(\mathcal{E})_{+}$is $(p, q)$-continuous on $\ell^{p}(\mathcal{E})_{+}$if it is $(p, q)$-stable on $\ell^{p}(\mathcal{E})_{+}$. The final corollary for this section shows how the stability condition can be relaxed in the presence of a stable finite-rank perturbation.

Corollary 2. Suppose that $C$ is causal and $T$ is $(p, q)$-stable on $\ell^{p}(\mathcal{E})_{+}$, where $\mathcal{E}$ is finite dimensional and $1 \leqslant p, q \leqslant \infty$. Suppose also that $T-C$ has finite rank, and that $C$ is $(p, q)$-stable on $\Xi_{+} \cap \Xi_{00}$. Then $C$ is $(p, q)$-continuous on $\ell^{p}(\mathcal{E})_{+}$.

Proof: Let $\mathcal{X}$ be the stability set of $C$, as defined in (1). By Theorem 1 it is enough to prove that $\mathcal{X}=\ell^{p}(\mathcal{E})_{+}$. According to the hypotheses of the corollary, $\Xi_{+} \cap \Xi_{00} \subseteq \mathcal{X}$.

Consider first the case $p<\infty$. Then $\Xi_{+} \cap \Xi_{00}$ is dense in $\ell^{p}(\mathcal{E})_{+}$and since $\mathcal{X}$ is closed, $\mathcal{X}=\ell^{p}(\mathcal{E})_{+}$. For the case $p=\infty$ we use the causality of $C$. Suppose that $x \in \ell^{\infty}(\mathcal{E})_{+}$. Then $P_{k} x \in \ell^{\infty}(\mathcal{E})_{+} \cap \Xi_{00}$, and so

$$
\left\|P_{k} C x\right\|_{q}=\left\|P_{k} C P_{k} x\right\|_{q} \leqslant\left\|C P_{k} x\right\|_{q} \leqslant \kappa\left\|P_{k} x\right\|_{\infty} \text { for each } k,
$$

where $\kappa$ is the $(\infty, q)$-operator norm of $C$ restricted to $\mathcal{X}$. Since $\left\|P_{k} x\right\|_{\infty} \rightarrow\|x\|_{\infty}$ and $\left\|P_{k} C x\right\|_{q} \rightarrow\|C x\|_{q}$ as $k \rightarrow \infty$, taking limits as $k \rightarrow \infty$ gives $\|C x\|_{q} \leqslant \kappa\|x\|_{\infty}$. Thus $C$ is $(\infty, q)$-continuous on $\ell^{\infty}(\mathcal{E})_{+}$.

\section{OPERATORS ON FINITE PAST AND TWO-SIDED SEQUENCES}

It is natural to look for results similar to Proposition 1 which apply to sequence space operators which are causal on other subspaces of $\ell^{p}(\mathcal{E})$, such as the whole space $\ell^{p}(\mathcal{E})$ or the subspace $\ell^{p}(\mathcal{E})_{f p}=\Xi_{f p} \cap \ell^{p}(\mathcal{E})$ of finite past sequences in $\ell^{p}(\mathcal{E})$. The following examples show that an operator may be $(p, q)$-stable and causal on these spaces without being $(p, q)$-continuous on the space. This extends a result obtained in [2] for the scalar-valued $(\infty, \infty)$-case.

EXAmple 2. There is a causal operator defined on $\Xi$ which, for each $1 \leqslant p, q \leqslant \infty$, is $(p, q)$-stable but not $(p, q)$-continuous.

Proof: To construct the desired operator $T$, first choose a Hamel basis $\mathcal{B}$ of $\Xi$ which contains a Hamel basis of $\Xi_{f p}$ and choose a sequence $x$ in $\ell^{1}(\mathcal{E})$ with infinite past. Let $T$ be the unique linear operator on $\Xi$ which satisfies

$$
T x=x \text { and } T z=0 \text { for each } z \in \mathcal{B} \backslash\{x\} .
$$

Since $x \in \ell^{q}(\mathcal{E})$ and the range of $T$ is spanned by $x, T$ is $(p, q)$-stable.

Now $T z=0$ for each vector $z$ in the Hamel basis of $\Xi_{f p}$, and so $T=0$ on $\Xi_{f p}$. Hence $T$ is causal because for any integer $k$ and any sequence $y$ in $\Xi,\left(1-P_{k}\right) y \in \Xi_{f p}$ and $P_{k} T\left(1-P_{k}\right) y=P_{k} 0=0$. 
Finally, since $x \in \ell^{1}(\mathcal{E}),\left\|P_{k} x\right\|_{p} \rightarrow 0$ as $k \rightarrow-\infty$. But $T P_{k} x=T x-$ $T\left(1-P_{k}\right) x=x \neq 0$, for each integer $k$, and so $T$ is not $(p, q)$-continuous.

EXAMPLE 3. There is a causal operator defined on $\Xi$ which, for each $1 \leqslant p, q \leqslant \infty$, is $(p, q)$-stable on $\Xi_{f p}$ but not $(p, q)$-continuous on $\Xi_{f p}$.

Proof: Let $T$ be the operator defined on $\Xi$ as follows: for each $x=\left(x_{n}\right)$ in $\Xi$,

$$
T x=y=\left(y_{n}\right), \text { where } y_{n}=2^{-n} x_{n} \text { for each } n .
$$

Since $T$ commutes with each projection $P_{k}, P_{k} T P_{k}=P_{k} T$ and so $T$ is causal.

To see that $T$ is $(p, q)$-stable on $\Xi_{f p}$ for each $1 \leqslant p, q \leqslant \infty$, choose $x=\left(x_{n}\right) \in$ $\ell^{p}(\mathcal{E})_{f p}$ and an integer $N$ such that $x_{n}=0$ for all $n<N$. Let $y=\left(y_{n}\right)=T x$. Then $y_{n}=0$ for $n<N$ and $\left|y_{n}\right| \leqslant 2^{-n}\left|x_{n}\right| \leqslant 2^{-n}\|x\|_{\infty}$ for $n \geqslant N$, and so

$$
\|y\|_{1} \leqslant \sum_{n \geqslant N} 2^{-n}\|x\|_{\infty}=2^{1-N}\|x\|_{\infty}<\infty
$$

So $T x \in \ell^{1}(\mathcal{E}) \subseteq \ell^{q}(\mathcal{E})$, and hence $T$ is $(p, q)$-stable on $\Xi_{f p}$.

On the other hand, $T$ is not $(p, q)$-continuous on $\Xi_{f p}$ because $\|T x\|_{q}=2^{-n}\|x\|_{p}$ if $x=\left(P_{n}-P_{n-1}\right) x$, and $2^{-n} \rightarrow \infty$ as $n \rightarrow-\infty$.

\section{SHIFT-COMMUTING OPERATORS}

The (forward) shift operator $S$ is defined on $\Xi$ by the formula:

for each $x=\left(x_{n}\right)$ in $\Xi, S x=y=\left(y_{n}\right)$, where $y_{n}=x_{n-1}$ for each $n$.

The shift $S$ is causal and an isometric isomorphism of each of the spaces $\ell^{p}(\mathcal{E})$ for $1 \leqslant p \leqslant \infty$. It intertwines the projections $P_{k}$ in the sense that

$$
S^{n} P_{k}=P_{k+n} S^{n} \text { for all integers } n \text { and } k \text {. }
$$

A set $\mathcal{M}$ of sequences is said to be shift-invariant if $x \in \mathcal{M}$ implies that $S x \in \mathcal{M}$. A linear form $\phi$ defined on a set of sequences is shift-invariant if its domain $\mathcal{D}(\phi)$ is shift-invariant, and for each $x$ in $\mathcal{D}(\phi), \phi(S x)=\phi(x)$.

For the remainder of this paper, we shall be concerned with sequence space operators which commute with the shift. In the signal processing literature, operators of this type are said to be shift-or time-invariant. We avoid this notation, preferring to reserve the term 'shift-invariance' for subsets of $\Xi$ and linear forms. 
4.1 ON CAUSAL SEqUenCEs. It follows immediately from Proposition 1 that a shiftcommuting linear operator $T$ which is causal and $(p, q)$-stable on $\Xi_{+}$, where $1 \leqslant p, q \leqslant$ $\infty$ and $\mathcal{E}$ is finite dimensional, is $(p, q)$-continuous on $\Xi_{+}$. In Theorem 3 we extend this result to the case where $\mathcal{E}$ is infinite dimensional.

THEOREM 3. If $T$ is causal, shift-commuting and $(p, q)$-stable on $\ell^{p}(\mathcal{E})_{+}$, where $1 \leqslant p, q \leqslant \infty$, then $T$ is $(p, q)$-continuous on $\Xi_{+}$.

Proof: The following is a 'gliding hump' argument of the type that can be used to establish basic automatic continuity results such as Proposition 1.

Suppose that $T$ is not $(p, q)$-continuous on $\Xi_{+}$. Then there exist sequences $x^{(n)}$ in $\ell^{p}(\mathcal{E})_{+}$such that for all positive integers $n$

$$
\left\|x^{(n)}\right\|_{p}<2^{-n} \text { and }\left\|T x^{(n)}\right\|_{q}>2^{n}+\sum_{j<n}\left\|T x^{(j)}\right\|_{q} .
$$

Choose positive integers $k_{1}, k_{2}, \ldots$ such that for all positive integers $n$,

$$
k_{n+1}>2 k_{n} \text { and }\left\|P_{k_{n}} T x^{(n)}\right\|_{q}>2^{n}+\sum_{j<n}\left\|T x^{(j)}\right\|_{q}
$$

and let $x=\sum_{j \geqslant 1} S^{k_{j}} x^{(n)}$. Then $x \in \ell^{p}(\mathcal{E})_{+}$and so $T x \in \ell^{q}(\mathcal{E})$.

Now let $m=2 k_{n}$. Since $T$ is causal,

$$
\begin{aligned}
P_{m} T x & =P_{m} T P_{m} x=P_{m} T P_{m}\left(\sum_{j \geqslant 1} S^{k_{j}} x^{(n)}\right) \\
& =P_{m} T P_{m}\left(\sum_{j=1}^{n} S^{k_{j}} x^{(n)}\right)=P_{m} T\left(\sum_{j=1}^{n} S^{k_{j}} x^{(n)}\right)
\end{aligned}
$$

and so

$$
\begin{aligned}
\left\|P_{m} T x\right\|_{q} & \geqslant\left\|P_{m} T S^{k_{n}} x^{(n)}\right\|_{q}-\sum_{j<n}\left\|P_{m} T S^{k_{j}} x^{(j)}\right\|_{q} \\
& \geqslant\left\|S^{k_{n}} P_{k_{n}} T x^{(n)}\right\|_{q}-\sum_{j<n}\left\|S^{k_{j}} T x^{(j)}\right\|_{q}>2^{n} .
\end{aligned}
$$

Hence $\|T x\|_{q}=\infty$, which is a contradiction, and $T$ must be $(p, q)$-continuous on $\Xi_{+}$.

A shift-commuting linear operator $T$ which maps causal sequences to causal sequences must be causal, since for any integer $k$,

$$
P_{k} T\left(1-P_{k}\right)=S^{k} P_{0} S^{-k} T S^{k}\left(1-P_{0}\right) S^{-k}=S^{k} P_{0} T\left(1-P_{0}\right) S^{-k}=0 .
$$

Hence we have the following corollary. 
Corollary 3. Suppose that $T$ is shift-commuting and maps causal sequences to causal sequences. If $T$ is $(p, q)$-stable on $\ell^{p}(\mathcal{E})_{+}$, where $1 \leqslant p, q \leqslant \infty$, then it is $(p, q)$-continuous on $\ell^{p}(\mathcal{E})_{+}$.

In Section 2.2 we extended the automatic continuity result of Proposition 1 to finite rank perturbations of causal operators on $\ell^{P}(\mathcal{E})_{+}$. There is a similar extension of Theorem 3 , with $q=\infty$ being an exceptional case.

Theorem 4. Suppose that $C$ is causal and $T$ is $(p, q)$-stable on $\ell^{p}(\mathcal{E})_{+}$, where $1 \leqslant p \leqslant \infty$ and $1 \leqslant q<\infty$. Suppose also that $T-C$ has finite rank, and that $T$ is shift-commuting. Then $T$ is causal and $(p, q)$-continuous on $\ell^{p}(\mathcal{E})_{+}$.

Proof: Suppose that $T$ is not causal on $\ell^{p}(\mathcal{E})_{+}$. Since $T$ is shift-commuting, this means that $\ell^{p}(\mathcal{E})_{+}$is not invariant under $T$, and there exists $x \in \ell^{p}(\mathcal{E})_{+}$such that $y=P_{0} T\left(1-P_{0}\right) x \neq 0$. Then $y \in \ell^{q}(\mathcal{E})$, and for any $n \geqslant 0$,

$$
S^{n} y=S^{n} P_{0} T\left(1-P_{0}\right) x=P_{n} T\left(1-P_{n}\right) S^{n} x=P_{n} f^{(n)},
$$

where $f^{(n)}=(T-C)\left(1-P_{n}\right) S^{n} x$.

Since $P_{n} f^{(n)}=S^{n} y,\left\|f^{(n)}\right\|_{q} \geqslant\|y\|_{q}>0$ for each integer $n \geqslant 0$. Furthermore, for each $j \leqslant n$,

$$
P_{j} f^{(n)}=P_{j} P_{n} f^{(n)}=P_{j} S^{n} y=S^{n} P_{j-n} y,
$$

and so $\left\|P_{j} f^{(n)}\right\|_{q}=\left\|P_{j-n} y\right\|_{q} \rightarrow 0$ as $n \rightarrow \infty$, since $q<\infty$.

Now let $e^{(n)}=f^{(n)} /\left\|f^{(n)}\right\|_{q}$. Then $\left(e^{(n)}\right)$ is a sequence of unit vectors in the finite dimensional range of $T-C$. So, by taking a subsequence if necessary, we may assume that $e^{(n)} \rightarrow e$ as $n \rightarrow \infty$, where $\|e\|_{q}=1$. For each integer $j$,

$$
\left\|P_{j} e^{(n)}\right\|_{q}=\left\|P_{j} f^{(n)}\right\|_{q} /\left\|f^{(n)}\right\|_{q} \leqslant\left\|P_{j} f^{(n)}\right\|_{q} /\|y\|_{q} \rightarrow 0
$$

as $n \rightarrow \infty$. So $P_{j} e=0$ for each integer $j$, and $e=0$, which is a contradiction. So we conclude that $y=0$ and $T$ is causal. Hence, by Theorem $3, T$ is $(p, q)$-continuous on $\ell^{p}(\mathcal{E})_{+}$.

To show that Theorem 4 does not extend to the case $q=\infty$, we first need to show that there are discontinuous, shift-invariant linear forms on each of the spaces $\ell^{P}(\mathcal{E})_{+}$. This is done by applying a well-known result concerning shift-invariant forms on the 'two-sided' scalar sequence spaces, $\ell^{p}(\mathbf{C})$.

LEMMA 1. For each $p$ in $[1, \infty]$ and each Banach space $\mathcal{E}$, there exists a discontinuous, shift-invariant linear form $\psi$ on $\ell^{p}(\mathcal{E})_{+}$with the property that $\psi=0$ on $\ell^{p}(\mathcal{E})_{+} \cap \Xi_{00}$

ProOF: By a result of Saeki [3] there are discontinuous shift-invariant forms $\phi_{1}$ and $\phi_{2}$ on $\ell^{p}(\mathrm{C})$, with the property that each non-zero linear combination of $\phi_{1}$ and $\phi_{2}$ 
is discontinuous. Let $\delta$ be a scalar sequence with just one non-zero term. If $\phi_{1}(\delta)=0$ let $\phi=\phi_{1}$; otherwise, let $\phi=\phi_{2}(\delta) \phi_{1}-\phi_{1}(\delta) \phi_{2}$. Then $\phi(\delta)=0$, and since $\phi$ is linear and shift-invariant, $\phi(x)=0$ for each $x$ in $\Xi_{00}(\mathbf{C})$.

Now let $\omega$ be a continuous non-zero linear form on $\mathcal{E}$. We can regard $\omega$ as a linear mapping from $\Xi(\mathcal{E})$ into $\Xi(\mathbf{C})$ by defining $\omega(x)=\left(\omega\left(x_{n}\right)\right)$ for each $\mathcal{E}$-valued sequence $x=\left(x_{n}\right)$. Then $\omega$ is shift-commuting, commutes with each projection $P_{k}$, and is continuous as a map from $\ell^{p}(\mathcal{E})$ into $\ell^{p}(\mathbf{C})$. Furthermore, the composite map $\eta=\phi \omega$ is a discontinuous, shift-invariant form on $\ell^{p}(\mathcal{E})$ with the property that $\eta=0$ on $\Xi_{00}(\mathcal{E})$.

Let $\eta_{+}$and $\eta_{-}$be the restrictions of $\eta$ to $\ell^{p}(\mathcal{E})_{+}$and to $\ell^{p}(\mathcal{E})_{-}=\ell^{p}(\mathcal{E})_{(-\infty, 0]}$ respectively. Since $\ell^{p}(\mathcal{E})_{+}$is shift-invariant, $\eta_{+}$is shift-invariant, and since $\eta=0$ on $\Xi_{00}(\mathcal{E}), \eta_{-}$is shift-invariant. Now let $\psi_{1}=\eta_{+}$and $\psi_{2}=\eta_{-} W$, where $W$ is the involution defined on $\Xi(\mathcal{E})$ by

$$
W x=y=\left(y_{n}\right), \text { where } y_{n}=x_{-n} \text { for each } n, \text { for each } x \text { in } \Xi(\mathcal{E}) .
$$

Both $\psi_{1}$ and $\psi_{2}$ are linear forms on $\ell^{p}(\mathcal{E})_{+}$, and $\psi_{1}$ is shift-invariant. To see that $\psi_{2}$ is also shift-invariant, note that for any $x$ in $\ell^{p}(\mathcal{E})_{+}$,

$$
\psi_{2}(S x)=\eta_{-} W(S x)=\eta_{-} S^{-1} W x=\eta_{-} W x=\psi_{2}(x) .
$$

Since $\eta$ is discontinuous, at least one of $\eta_{+}$and $\eta_{-}$is discontinuous, and so at least one of $\psi_{1}$ and $\psi_{2}$ has the desired properties.

This lemma can be used to show that Theorem 4 does not extend to the case $q=\infty$.

EXAMPLE 4. There is a shift-commuting rank one operator which is $(p, \infty)$-stable, but not $(p, \infty)$-continuous on $\ell^{p}(\mathcal{E})_{+}$, for each $1 \leqslant p \leqslant \infty$.

Proof: Let $T x=\psi(x) e$ for each $x$ in $\ell^{p}(\mathcal{E})_{+}$, where $1 \leqslant p \leqslant \infty$, where $\psi$ is a discontinuous shift-invariant linear form on $\ell^{p}(\mathcal{E})_{+}$, and where $e$ is a non-zero constant $\mathcal{E}$-valued sequence. Clearly $T$ has rank one and is $(p, \infty)$-stable, but not $(p, \infty)$-continuous on $\ell^{p}(\mathcal{E})_{+}$. Finally, $T$ is shift-commuting since

$$
S T x=\psi(x) S e=\psi(x) e=\psi(S x) e=T S x \text { for each } x \text { in } \ell^{p}(\mathcal{E})_{+} .
$$

The zero operator is a causal finite-rank perturbation of $T$ which shows that Theorem 4 does not extend to the case $q=\infty$.

\subsection{ON FINITE PAST SEQUENCES.}

The isometric nature of the shift operator can be used to extend the automatic continuity results for operators on causal sequences to shift-commuting operators on 
finite past sequences. Thus, for example, if $T$ is shift commuting and $x \in \ell^{p}(\mathcal{E})_{f p}$, then $S^{k} x \in \ell^{p}(\mathcal{E})_{+}$for some integer $k$, and so

$$
\|T x\|_{q}=\left\|S^{k} T x\right\|_{q}=\left\|T S^{k} x\right\|_{q} .
$$

So $T$ is $(p, q)$-stable on $\ell^{p}(\mathcal{E})_{f p}$ if and only if $T$ is $(p, q)$-stable on $\ell^{p}(\mathcal{E})_{f_{p}}$.

LEMma 2. Suppose that $T$ is shift-commuting on $\ell^{p}(\mathcal{E})_{f p}$ and is $(p, q)$-continuous on $\ell^{p}(\mathcal{E})_{+}$, where $1 \leqslant p, q \leqslant \infty$. Then $T$ is $(p, q)$-continuous on $\ell^{p}(\mathcal{E})_{f p}$.

PRoof: If $x \in \ell^{p}(\mathcal{E})_{f p}$ then $S^{k} x \in \ell^{p}(\mathcal{E})_{+}$for some integer $k$, and

$$
\|T x\|_{q}=\left\|S^{k} T x\right\|_{q}=\left\|T S^{k} x\right\|_{q} \leqslant \kappa\left\|S^{k} x\right\|_{p}=\kappa\|x\|_{p},
$$

where $\kappa$ is the $(p, q)$-norm of $T$ restricted to $\ell^{p}(\mathcal{E})_{f_{p}}$.

The first corollary, which is an analogue of Proposition 1 for shift-commuting, causal operators on $\ell^{p}(\mathcal{E})_{f p}$, is an immediate consequence of Lemma 2 and Theorem 3 . The second corollary, which is an analogue of Theorem 2 , follows immediately from Lemma 2 and Theorem 4.

COROLLARY 4. If $T$ is shift-commuting, causal and $(p, q)$-stable on $\ell^{p}(\mathcal{E})_{f p}$, where $1 \leqslant p, q \leqslant \infty$, then it is $(p, q)$-continuous on $\ell^{P}(\mathcal{E})_{f p}$.

COROLlaRY 5. Suppose that $T$ is a finite rank perturbation of a causal operator which is shift-commuting and $(p, q)$-stable on $\ell^{p}(\mathcal{E})_{f p}$, where $1 \leqslant p \leqslant \infty$ and $1 \leqslant q<$ $\infty$. Then $T$ is $(p, q)$-continuous on $\ell^{p}(\mathcal{E})_{f p}$.

\subsection{ON TWO-SIDED SEQUENCES.}

There is a well-established automatic continuity theory for shift-commuting sequence space operators. It follows from a result of Johnson [1] that there are shiftcommuting operators which are $(p, q)$-stable, but not $(p, q)$-continuous if and only if there is a discontinuous rank-one operator $F$ on $\ell^{p}(\mathcal{E})$ of the form

$$
F x=\psi(x) y \text { for each } x \text { in } \ell^{p}(\mathcal{E}),
$$

where $\psi$ is a discontinuous shift-invariant form on $\ell^{p}(\mathcal{E})$, and where $y$ is a non-zero vector in $\ell^{q}(\mathcal{E})$ for which $y=S y$.

However, only $\ell^{\infty}(\mathcal{E})$ contains vectors $y$ of this type, and so each shift-commuting $(p, q)$-stable operator is $(p, q)$-continuous if $q<\infty$. On the other hand, it follows from a result of Saeki [3] that there are non-zero discontinuous shift-invariant forms on $\ell^{p}(\mathcal{E})$ for each $p$ in $[1, \infty]$. So for each such $p$ there are shift-commuting operators which are $(p, \infty)$-stable but not $(p, \infty)$-continuous. Our final example shows that there is a discontinuous $(p, \infty)$-stable operator which is both shift-commuting and causal. 
EXample 5. There is a rank-one, shift-commuting causal operator on $\ell^{p}(\mathcal{E})$, where $1 \leqslant p \leqslant \infty$, which is $(p, \infty)$-stable but not $(p, \infty)$-continuous.

Proof: Let $\psi$ be a discontinuous, shift-invariant linear form on $\ell^{P}(\mathcal{E})_{+}$, with the property that $\psi=0$ on $\ell^{p}(\mathcal{E})_{+} \cap \Xi_{00}$, and let $\Psi=\psi\left(1-P_{0}\right)$. Then $\Psi$ is a discontinuous linear form on $\ell^{p}(\mathcal{E})$ and $\Psi P_{j}=0$ for each integer $j$. Furthermore, $\Psi$ is shift-invariant since

$$
\Psi S=\Psi\left(1-P_{1}\right) S=\psi\left(1-P_{1}\right) S=\psi S\left(1-P_{0}\right)=\psi\left(1-P_{0}\right)=\Psi .
$$

Now let $\Phi=\Psi W$, where $W$ is the involution introduced in the proof of Lemma 1 , and let $F \boldsymbol{x}=\Phi(x) y$, where $y$ is a non-zero constant $\mathcal{E}$-valued sequence. Clearly $F$ is a rank-one operator which is $(p, \infty)$-stable but not $(p, \infty)$-continuous. It is shiftcommuting because $\psi$ is shift-invariant and so

$$
\Phi S=\Psi W S=\Psi S W S=\Psi W S^{-1} S=\Psi W=\Phi .
$$

Finally, $F$ is causal. To see this, we observe that for each integer $j$,

$$
\Phi=\Psi W=\Psi\left(1-P_{-j-1}\right) W=\Psi W P_{j}=\Phi P_{j},
$$

and so for each $x$ in $\ell^{p}(\mathcal{E})$,

$$
P_{j} F\left(1-P_{j}\right) x=\Phi\left(\left(1-P_{j}\right) x\right) P_{j} y=0 .
$$

\section{REFERENCES}

[1] B.E. Johnson, 'Continuity of homomorphisms of Banach G-modules', Pacific J. Math. 120 (1985), 111-121.

[2] L.T. Ramsey, 'Bounded-in-bounded-out, linear, causal operators on causal sequences have finite gain', (preprint).

[3] S. Saeki, 'Discontinuous translation invariant functionals', Trans. Amer. Math. Soc. 282 (1984), 403-414.

[4] A.M. Sinclair, Automatic continuity of linear operators (Cambridge University Press, 1976).

\footnotetext{
Division of Science

Murdoch University

Murdoch WA 6150

Australia

e-mail: harrison@prodigal.murdoch.edu.au

L.Eaton@math.canterbury.ac.nz

ward@prodigal.murdoch.edu.au
} 